\title{
Epidemiology of Musculoskeletal Disorders in Musicians - Systematic Review
}

\author{
Ketaki Vijay Shinde ${ }^{1}$, Pradeep Borkar ${ }^{2}$ \\ ${ }^{1}$ Intern, Dr. A.P.J. Abdul Kalam College of Physiotherapy, Pravara Institute of Medical Sciences, \\ 'Deemed to be' University, Loni, Maharashtra, India \\ ${ }^{2}$ Associate Professor, Orthopedic Physiotherapy Department, Dr. APJ Abdul Kalam College of Physiotherapy, \\ Pravara Institute of Medical Sciences, ‘Deemed to be’ University, Loni, Maharashtra, India
}

Corresponding Author: Ketaki Vijay Shinde

\begin{abstract}
Musicians performing over an instrument demands repetitive, awkward postures and postural stress from prolonged sitting or standing. Musicians are found to be more prone to the development of Playing-Related Musculoskeletal Disorders (PRMDs) due to these factors. PRMDs are defined as 'pain, weakness, numbness, tingling or other symptoms that interfere with their ability to play an instrument'. There is an increasing interest seen in the medical issues faced by the musicians performing over an instrument. There are different type of musicians playing different instruments which means different instrumentalists face different musculoskeletal disorders depending upon many factors. There are intrinsic and extrinsic factors involved which contribute to the development of PRMDs in musicians. Development of PRMDs is seen to be a cause of early termination of musician's career. The purpose of study is to review the epidemiology of musculoskeletal disorders in musicians.
\end{abstract}

Key Words: Musculoskeletal disorders, Musculoskeletal problems, Prevalence of musculoskeletal injuries, Postural disorders, Playing-related strain in instrumentalists and musicians.

\section{INTRODUCTION}

A musician is a person who performs music. The term musician is used for a person who follows performing music as a profession. Musicians may perform on their own or as a part of group, band or orchestra. There are different types of musicians playing different instruments percussionist, string musicians, pianist, wind musicians.

There is an increasing interest seen in medical conditions of performing artists in last 20 years ${ }^{1}$. Major Playing Related Musculoskeletal Disorders are to be found in musicians ${ }^{2}$. Earlier musicians were advised about "No pain, no gain" in order to achieve performance skills and excellence ${ }^{2}$. Though this advice was well intended, but it has proved to be responsible for putting the musicians at a high risk of developing musculoskeletal disorders, eventually career ending, also called as Playing Related Musculoskeletal Disorders (PRMD's) or pain (PRP - Playing Related Pain) ${ }^{3}$. Pain and first symptoms of overloads are often underestimated by musicians in early stages sent by the body ${ }^{3}$. A bit of studies disclosed that musicians consider treating pain and disorders caused due to playing an instrument as an indication of fragility and inability $^{3}$. Regrettably this perspective causes high incidence of impairment and expulsion from their profession ${ }^{3}$.

Performing music over an instrument demands repetitive, awkward postures while playing and postural stress from prolonged sitting or standing and transporting instruments, music stands, 
microphones, speakers and other equipment's by the musician ${ }^{4}$. Relying on type of instrument, the musician has to attain an uneven posture (e.g. - violin, viola, flute, guitar) and even posture (e.g. - piano) and can play with persistently elevated upper limbs (e.g. - violin, flute) or not (e.g. - drums, piano $)^{3}$. Even countless hours of practice contribute in enlargement of tissue damage $^{3}$. The stress of upcoming concerts and contests contributes to tension and intensifies the condition ${ }^{3}$. These factors that are required for performing music over an instrument causes musicians to have high susceptibility of developing musculoskeletal problems like pain, overuse injuries, focal dystonia, nerve entrapment and peripheral neuropathies ${ }^{5}$. Most common symptom of overuse among instrumentalists is found to be pain and most commonly affected are the string players ${ }^{4}$. Set of instruments that lead to frequent occurrence of pain are string instruments and wind instruments ${ }^{4}$.

Musician career is endangered when they are no longer able to play instrument and can have undesirable impact on their economy because of musculoskeletal disorders as they need to take rest to heal from injuries ${ }^{6}$. It was shown in an overview of literature, musculoskeletal disorders more affected women than men ${ }^{6}$. It was found in the national survey of orchestral musician's that $76 \%$ of participants took off from performing due to occurrence of serious injury in their career ${ }^{6}$. It was stated by some small studies that $50 \%$ of treated musicians were unable to return back to their career of performing music 6 . According to the Bureau of Labour Statistics, approximately 264,000 musicians were found to be employed in United States in $2006^{6}$. Among them 50\% to $76 \%$ of professional musician's reported occurrence of musculoskeletal injuries ${ }^{6}$. In a University of Texas study, it was stated that prevalence of musculoskeletal injuries amidst the brass instrument players (French horn, trombone, trumpet or tuba) was $61 \%{ }^{6}$. Highest rate of injuries was found in female trombonists concerning left upper extremity and upper back ${ }^{6}$. A study conducted by
International Conference of Symphony and Opera musicians found that $70 \%$ of women and $52 \%$ of men reported incidence of Playing Related Musculoskeletal Disorders ${ }^{6}$. Amidst 1353 instrumentalist evaluated, major diagnoses included musculoskeletal disorders in 64\%, peripheral nerve problems in $20 \%$ and focal dystonia in $8 \%{ }^{1}$. Musculoskeletal disorders that were found included regional muscle pain syndrome in which upper limb, upper trunk and neck were most common ${ }^{1}$. Thoracic outlet syndrome, ulnar neuropathy at elbow and carpal tunnel syndrome ${ }^{1}$. Musicians playing violin or viola showed four times expansion in right forearm pain and twice risk for cervical pain, right shoulder pain and left forearm pain so as differentiated with pianists ${ }^{4}$. The least affected with playing-related musculoskeletal disorders are percussion musicians ${ }^{4}$. High rate of disorders is corelated with piano, guitar and harp ${ }^{4}$. Bilateral ulnar deviation is seen more in the wrist postures playing piano than in any other instrument ${ }^{4}$.

Playing Related Musculoskeletal Disorders are also caused due to issues involved in the interface between a musician and the instrument ${ }^{4}$. Intrinsic and extrinsic type of factors are involved ${ }^{4}$. Intrinsic factors for PRMD's consist of musician's size, strength, muscle tone, flexibility and existence of primary disorder ${ }^{4}$. Physical training is not highlighted in music schools which leads to development of risk factors for further life-threatening health conditions ${ }^{4}$. Musicians frequently spend time practicing for hours as differentiated with athletes ${ }^{4}$. Poor physical fitness contributes in occurrence of musculoskeletal disorders ${ }^{4}$. Extrinsic factors for PRMD's consist of the environment in which a musician performs and the technique used by the musician to play an instrument ${ }^{4}$. Techniques by which a musician plays an instrument involves the way a musician holds the instruments, force used to play an instrument and the occurrence of awkward and static or dynamic loading postures ${ }^{4}$. 
Most musical instruments demand maintenance of awkward posture for prolonged period of time which is difficult to neglect ${ }^{7}$. Posture plays an important role in maintaining body tissue integrity ${ }^{7}$. Ergonomically incorrect posture can lead to numerous musculoskeletal disorders ${ }^{7}$. Example - increased tissue loading is seen in slumped posture with altered movement behaviour ${ }^{7}$. Posture change has consequences for both risk of injury and musical performance ${ }^{7}$.

Static load causes prolonged muscle contraction and tension across the joint and supportive soft tissue and bony structures ${ }^{4}$. Occurrence of static load is seen when an instrument is held in a fairly immobile position $^{4}$. In divergence, dynamic load is force on joints, muscles and supportive structures ${ }^{4}$. There should be ideal balance between static load and dynamic load if aiming to reduce static load and increasing dynamic loads on connected joints and structures while playing ${ }^{4}$ Playing environment comprises of proper organization of work and break periods ${ }^{4}$. A hasty increase in practice session time can lead to increased strain on the associated joints and eventual incidence of pain and fatigue, consequently leading to occurrence of musculoskeletal disorders ${ }^{4}$. It was reported in a study that higher incidence of musculoskeletal disorders was found in musicians who practice for more than 20 hours per week ${ }^{4}$. Previously, many other researchers have stated that due to prolonged awkward posture while playing and prolonged static posture can lead to musculoskeletal disorders in musicians ${ }^{4}$.

Aim: To review the epidemiology of musculoskeletal disorders in Musicians.

Objective: To review the epidemiology of musculoskeletal disorders in Musicians.

\section{MATERIALS AND METHOD}

The aim was to study and summarize the findings in the articles on epidemiology of musculoskeletal disorders that occur in musicians. This review was carried out by searching in databases including PubMed,
Google Scholar, ResearchGate, Science Direct, sciELO. Search was done using the following keywords Musculoskeletal disorders, Musculoskeletal problems, Prevalence of musculoskeletal injuries, Postural disorders, Playing-related strain in instrumentalists and musicians. Articles included in the study were between January 2011-December 2019 and the study was carried out in Pravara Institute of Medical Sciences. The inclusion criteria were, 1) Full text articles, 2) Articles which are published in last 10 years, 3) Crosssectional studies and observational studies, 4) Population including population of all genders, 5) Population with age group ranging between $18-79$ years. The exclusion criteria were as follows: 1) Duplicate articles, 2) Case reports, 3) Articles with only abstracts, 4) Statistics which did not present $\%$ of specific disorders.

\section{Search Strategy:}

The following databases were searched: PubMed, Google Scholar, sciELO, Science Direct and Research Gate between January 2011- December 2019. In addition, other sources and reference lists of published papers were searched. The search keywords utilized were musicians; musculoskeletal disorders; musculoskeletal problems; prevalence of musculoskeletal injuries; postural disorders and playingrelated strain in instrumentalists and musicians.

\section{Study Selection:}

The preliminary search returned 84 published abstracts. Then 75 potentially relevant papers were identified and 9 duplicates were excluded. The second stage of the selection strategy involved the examination of each of the 75 screened abstracts and 20 articles were excluded due to inadequacy of statistical evaluation. In the next stage, 55 full text articles were assessed according to the eligibility criteria and 29 studies were excluded. Finally, 26 articles were selected for methodological quality assessment. 7 studies that 
accomplished $\leq 9$ points according to the QUADAS tool were excluded after the methodological quality assessment. Finally, 19 studies were included in the qualitative synthesis.

\section{Data Extraction:}

The following information was extracted: author's name, publication year, sample size, description of data collection methods, prevalence rate, response rate, outcomes and associated factors related to PRMD.

\section{Procedure:}

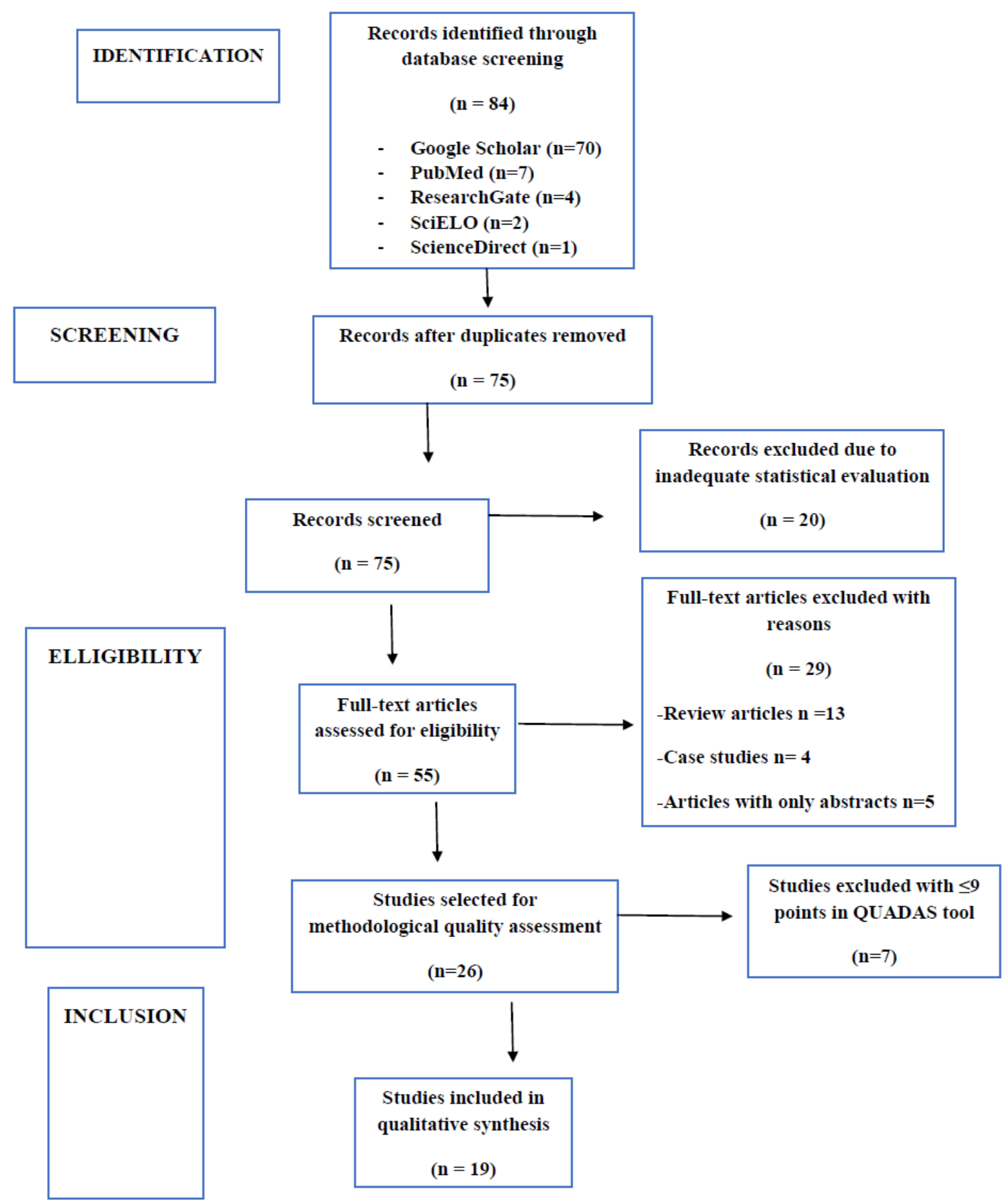

Figure 1- PRISMA flow diagram 
Assessment of Methodological Quality:

The assessment of methodological quality was performed using QUADAS tool (Table 1). Whiting P, Rutjes AW, Reitsma $\mathrm{JB}$, et.al established the development of QUADAS: a tool for the quality assessment of studies of diagnostic accuracy included in systematic reviews which was recommended by Cochrane Collaboration. This scale consisted of 7 questions concerning the sample size, presence of inclusion and exclusion criteria, use of standard assessment method, type of samples studied (professional musicians or music students), presence of conflict of interest and presence of consent of local bioethics commission. The maximum score possible was 14 and the studies were classified as low evidential value (0-8 points), moderate evidential value (9-11 points) and high evidential value (12-14 points). Methodological Quality assessment of articles according to QUADAS tool was carried out (Table 2).

\begin{tabular}{|l|l|l|}
\hline Table 1. QUADAS tool: quality assessment of studies of diagnostic accuracy included in systematic reviews. \\
\hline Q. No. & QUESTIONS AND PUNCTUATIONS & \\
\hline Q1. & The size of the studied group & $\begin{array}{l}0-9-0 \text { pts. } \\
10-99-1 \text { pt. } \\
\text { More than } 100-2 \text { pts. }\end{array}$ \\
\hline Q2. & Presence of exclusion criteria & $\begin{array}{l}\text { None }-0 \text { pts. } \\
\text { Present }-2 \text { pts. }\end{array}$ \\
\hline Q3. & Presence of inclusion criteria & $\begin{array}{l}\text { None }-0 \text { pts. } \\
\text { Present }-2 \text { pts. }\end{array}$ \\
\hline Q4. & Standard assessment of ailments & $\begin{array}{l}\text { Other/No information }-0 \text { pts. } \\
\text { Interview/Questionnaire }-1 \text { pt. } \\
\text { Standard Questionnaire }-2 \text { pts. }\end{array}$ \\
\hline Q5. & The group studied professional musicians & $\begin{array}{l}\text { No information }-0 \text { pts. } \\
\text { University students/School students }-1 \text { pt. } \\
\text { Professionals }-2 \text { pts. }\end{array}$ \\
\hline Q6. & Presence of a conflict of interest & $\begin{array}{l}\text { Present }-0 \text { pts. } \\
\text { No information }-1 \text { pt. } \\
\text { None }-2 \text { pts. }\end{array}$ \\
& & $\begin{array}{l}\text { No information }-0 \text { pts. } \\
\text { Present }-2 \text { pts. }\end{array}$ \\
\hline Q7. & The presence of the consent of the local bioethics commission \\
\hline & $\begin{array}{l}\text { Punctuations: } \\
\text { 9-8 - low evidential value } \\
12-14-\text { hoderate evidential value }\end{array}$ & \\
\hline
\end{tabular}

\begin{tabular}{|c|c|c|c|c|c|c|c|c|c|c|}
\hline \multirow{2}{*}{$\begin{array}{l}\text { Sr } \\
\text { No. }\end{array}$} & \multirow{2}{*}{$\begin{array}{l}\text { Author of the Article } \\
\text { and Year of } \\
\text { Publication }\end{array}$} & \multirow{2}{*}{ Title of the article } & \multicolumn{7}{|c|}{ Punctuations } & \multirow{2}{*}{ Result } \\
\hline & & & 1 & 2 & 3 & 4 & 5 & 6 & 7 & \\
\hline 1. & $\begin{array}{l}\text { A. Steinmetz et.al } \\
2012\end{array}$ & $\begin{array}{l}\text { Playing-related musculoskeletal disorders in music } \\
\text { students-associated musculoskeletal signs }\end{array}$ & 1 & 2 & 2 & 1 & 1 & 2 & 0 & $\begin{array}{l}9 \\
\text { (moderate) }\end{array}$ \\
\hline 2. & $\begin{array}{l}\text { Flavio M. Silva, PT, } \\
\text { ScD et.al } \\
2018\end{array}$ & $\begin{array}{l}\text { Musicians injuries: upper quarter motor control deficits in } \\
\text { musicians with prolonged symptoms- a case control study }\end{array}$ & 1 & 2 & 2 & 1 & 2 & 1 & 0 & $\begin{array}{l}9 \\
\text { (moderate) }\end{array}$ \\
\hline 3. & $\begin{array}{lll}\text { Dianna Kenny et.al. } \\
2013\end{array}$ & $\begin{array}{l}\text { Performance-related musculoskeletal pain, depression and } \\
\text { music performance anxiety in professional orchestral } \\
\text { musicians: a population study }\end{array}$ & 2 & 2 & 2 & 2 & 2 & 0 & 2 & $\begin{array}{l}12 \\
\text { (high) }\end{array}$ \\
\hline 4. & $\begin{array}{l}\text { Filiz Ozdemir et.al } \\
2019\end{array}$ & $\begin{array}{l}\text { Evaluation of work-related musculoskeletal disorders and } \\
\text { ergonomic risk levels among instrumentalist musicians }\end{array}$ & 1 & 2 & 2 & 2 & 2 & 0 & 2 & $\begin{array}{l}11 \\
\text { (moderate) }\end{array}$ \\
\hline 5. & $\begin{array}{l}\text { Chia-Ying Ling et.al. } \\
2018\end{array}$ & $\begin{array}{l}\text { Playing-related musculoskeletal disorders among classical } \\
\text { piano students at tertiary institutions in Malaysia }\end{array}$ & 2 & 2 & 2 & 1 & 2 & 2 & 2 & $\begin{array}{l}8 \\
\text { (low) }\end{array}$ \\
\hline 6. & $\begin{array}{l}\text { Adedayo T. Ajidahun } \\
\text { et.al. } 2017\end{array}$ & $\begin{array}{l}\text { Musculoskeletal problems among string instrumentalist in } \\
\text { South Africa }\end{array}$ & 2 & 2 & 2 & 1 & 2 & 0 & 2 & $\begin{array}{l}11 \\
\text { (moderate) }\end{array}$ \\
\hline 7. & $\begin{array}{l}\text { P. Sathya et.al } \\
2019\end{array}$ & $\begin{array}{l}\text { A study to find prevalence of upper limb problems in } \\
\text { musicians }\end{array}$ & 2 & 2 & 2 & 1 & 1 & 0 & 0 & $\begin{array}{l}8 \\
\text { (low) }\end{array}$ \\
\hline 8. & $\begin{array}{l}\text { Sagar Vedpathak et.al } \\
2017\end{array}$ & $\begin{array}{l}\text { Common musculoskeletal injuries faced by Indian } \\
\text { drummers }\end{array}$ & 1 & 2 & 2 & 1 & 2 & 0 & 0 & $\begin{array}{l}8 \\
\text { (low) }\end{array}$ \\
\hline 9. & $\begin{array}{l}\text { E. Savino et.al } \\
2013\end{array}$ & $\begin{array}{l}\text { Musculoskeletal disorders and occupational stress of } \\
\text { violinists }\end{array}$ & 1 & 2 & 2 & 2 & 1 & 2 & 0 & 10 \\
\hline 10. & $\begin{array}{l}\text { Helene M Paarup et.al } \\
2011\end{array}$ & $\begin{array}{l}\text { Prevalence and consequences of musculoskeletal } \\
\text { symptoms in symphony orchestra musicians vary by } \\
\text { gender: a cross-sectional study }\end{array}$ & 2 & 2 & 0 & 2 & 2 & 0 & 2 & $\begin{array}{l}10 \\
\text { (moderate) }\end{array}$ \\
\hline
\end{tabular}




\begin{tabular}{|c|c|c|c|c|c|c|c|c|c|c|}
\hline \multicolumn{11}{|c|}{ Table 2 Continued... } \\
\hline 11. & $\begin{array}{l}\text { Helene M Paarup et.al } \\
2012\end{array}$ & $\begin{array}{l}\text { Occurrence and co-existence of musculoskeletal symptoms } \\
\text { and findings in work-attending orchestra musicians - an } \\
\text { exploratory cross-sectional study }\end{array}$ & 2 & 2 & 2 & 0 & 2 & 2 & 0 & $\begin{array}{l}10 \\
\text { (moderate) }\end{array}$ \\
\hline 12. & $\begin{array}{l}\text { Katriina Viljamaa et.al } \\
2017\end{array}$ & $\begin{array}{lllll}\begin{array}{l}\text { Musculoskeletal symptoms } \\
\text { musicians }\end{array} & \text { among finish orchestral } \\
\end{array}$ & 2 & 2 & 2 & 2 & 2 & 0 & 2 & (high) \\
\hline 13. & $\begin{array}{ll}\text { Érico Felden Pereira } \\
\text { et.al. } \\
2014\end{array}$ & $\begin{array}{l}\text { Work related stress and musculoskeletal complaints of } \\
\text { orchestra musicians }\end{array}$ & 1 & 2 & 0 & 2 & 2 & 2 & 2 & $\begin{array}{l}8 \\
\text { (low) }\end{array}$ \\
\hline 14. & $\begin{array}{l}\text { Laura M Kok et.al. } \\
2013\end{array}$ & $\begin{array}{l}\text { A comparative study on the prevalence of musculoskeletal } \\
\text { complaints among musicians and non-musicians }\end{array}$ & 2 & 2 & 2 & 1 & 1 & 0 & 2 & $\begin{array}{l}10 \\
\text { (moderate) }\end{array}$ \\
\hline 15. & $\begin{array}{l}\text { Dusica L. Maric et.al } \\
2019\end{array}$ & $\begin{array}{l}\text { A painful symphony: the presence of overuse syndrome in } \\
\text { professional classical musicians }\end{array}$ & 1 & 2 & 2 & 1 & 2 & 0 & 2 & $\begin{array}{l}10 \\
\text { (moderate) }\end{array}$ \\
\hline 16. & $\begin{array}{l}\text { Y. Kaufman-Cohen } \\
\text { et.al. } 2011\end{array}$ & $\begin{array}{l}\text { Correlation between risk factors and musculoskeletal } \\
\text { disorders among classical musicians }\end{array}$ & 1 & 2 & 2 & 2 & 2 & 2 & 2 & $\begin{array}{l}13 \\
\text { (high) }\end{array}$ \\
\hline 17. & $\begin{array}{l}\text { A. Steinmetz et.al. } \\
2015\end{array}$ & $\begin{array}{l}\text { Frequency, severity and predictors of playing-related } \\
\text { musculoskeletal pain in professional orchestral musicians } \\
\text { in Germany }\end{array}$ & 2 & 2 & 0 & 2 & 2 & 0 & 2 & (moderate) \\
\hline 18. & $\begin{array}{l}\text { Adedayo } \quad \text { Tunde } \\
\text { Ajidahun et.al. } 2016\end{array}$ & $\begin{array}{l}\text { Upper extremity disability among string instrumentalists- } \\
\text { use of the quick dash and the ndi }\end{array}$ & 1 & 2 & 0 & 2 & 2 & 0 & 2 & $\begin{array}{l}9 \\
\text { (moderate) }\end{array}$ \\
\hline 19. & $\begin{array}{l}\text { Yael Kaufman-Cohen } \\
\text { et.al. } 2018\end{array}$ & $\begin{array}{l}\text { The correlation between upper extremity musculoskeletal } \\
\text { symptoms and joint kinematics, playing habits and hand } \\
\text { span during playing among piano students }\end{array}$ & 1 & 2 & 2 & 1 & 1 & 0 & 1 & $\begin{array}{l}8 \\
\text { (low) }\end{array}$ \\
\hline 20. & $\begin{array}{ll}\text { Frederico } & \text { Baretto } \\
\text { Kochem et.al } & \\
2017 & \\
\end{array}$ & $\begin{array}{l}\text { Prevalence and associated factors of playing-related } \\
\text { musculoskeletal disorders in brazilian violin players }\end{array}$ & 2 & 2 & 2 & 2 & 2 & 2 & 2 & $\begin{array}{l}14 \\
\text { (high) }\end{array}$ \\
\hline 21. & $\begin{array}{l}\text { Heiner Gembris et.al } \\
2017\end{array}$ & $\begin{array}{l}\text { Health problems of orchestral musicians from a life span } \\
\text { perspective: results of a large-scale study }\end{array}$ & 2 & 2 & 0 & 2 & 1 & 0 & 2 & $\begin{array}{l}9 \\
\text { (moderate) }\end{array}$ \\
\hline 22. & $\begin{array}{l}\text { Cláudia Maria SOUSA } \\
\text { et.al. } 2017\end{array}$ & $\begin{array}{l}\text { Playing-related musculoskeletal disorders of professional } \\
\text { orchestra musicians from the north of portugal: comparing } \\
\text { string and wind musicians }\end{array}$ & 2 & 2 & 0 & 1 & 2 & 2 & 2 & 11 \\
\hline 23. & $\begin{array}{l}\text { Laura M. Kok et.al. } \\
2016\end{array}$ & $\begin{array}{l}\text { The influence of sudden increase in playing time on } \\
\text { playing-related musculoskeletal complaints in high-level } \\
\text { amateur musicians in a longitudinal cohort study }\end{array}$ & 1 & 2 & 2 & 2 & 2 & 1 & 0 & $\begin{array}{l}8 \\
\text { (low) }\end{array}$ \\
\hline 24. & $\begin{array}{l}\text { Luis Heredia et.al. } \\
2014\end{array}$ & $\begin{array}{l}\text { Playing-related musculoskeletal problems among } \\
\text { musicians of orquesta buena vista social club and } \\
\text { supporting bands }\end{array}$ & 1 & 2 & 0 & 2 & 2 & 0 & 0 & $\begin{array}{ll}7 \\
\text { (low) }\end{array}$ \\
\hline 25. & $\begin{array}{llr}\text { Mark } & \text { Porter et.al. } \\
2018 & & \end{array}$ & $\begin{array}{l}\text { Extent of playing-related musculoskeletal problems in irish } \\
\text { traditional music community a survey }\end{array}$ & 1 & 2 & 0 & 2 & 1 & 2 & 2 & $\begin{array}{l}10 \\
\text { (moderate) }\end{array}$ \\
\hline 26. & R. Leaver 2011 & $\begin{array}{l}\text { Musculoskeletal pain in elite professional musicians from } \\
\text { british symphony orchestras }\end{array}$ & 2 & 2 & 2 & 2 & 2 & 2 & 0 & 12 \\
\hline
\end{tabular}

\section{RESULT}

The studies methodological quality was shown to be of moderate evidential value. Fourteen of 26 assessed articles using the QUADAS tool were classified as of moderate evidential value, seven studies as low evidential value and five studies as high evidential value. The main methodological faults according to the QUADAS tool were absence of specificity of exclusion criteria used in sample selection followed by inadequate information about conflicts of interest and presence of consent.

The sample size of included articles ranged from 12 to 2536 music professionals and music students. These subjects reported ages ranging from 18 to 79 years of age. Total number of males included in the study were $n=3050$ and total number of females were $\mathrm{n}=2737$. Most studies included orchestral musicians 11,14,22-24,29. Eight papers included the string instrumentalists $18,26-29,33,37$ and some studies included combination of different instrumentalists like percussionists, wind musicians, keyboardists, trombone players, brass players $9,13,26-28,37$. One paper also included fiddle players ${ }^{36}$. Two studies included violinists alone ${ }^{21,32}$.

\section{Sociodemographic factors: Gender-}

Effect of gender as a determinant for musculoskeletal disorders has been frequently examined 11,14,18,22,24,28,29,32. In multivariate analysis in the cross-sectional study, it was found that females were most likely to experience musculoskeletal symptoms like pain, disability at early stage than their male colleagues. Also, females presented greater risk to develop musculoskeletal disabilities ${ }^{32}$. 
multivariate study presented, female musicians reported significantly more often incidences of pain that affected with their performance $^{11}$. The prevalence rate of PRMD was measured during different times and there was a significant association found between being a female gender and musculoskeletal symptoms in at least one anatomic region ${ }^{22}$. The prevalence of symptom in one anatomic region within twelve months was reported by $97 \%$ of women and $83 \%$ of men. Seven days or more of symptoms were experienced by $86 \%$ of women and $67 \%$ of men and $63 \%$ of women and $49 \%$ of men had problems for more than 30 days within a year ${ }^{22}$. A study showed difference between anatomic regions where the musculoskeletal symptoms were experienced by female and male professional orchestra musicians. The study states that female musicians experienced significantly more neck, elbow and wrist pain than their male colleagues. However, analysis of results of papers showed that there is positive association between gender and musculoskeletal disorders.

Age-

Co-relation between age and musculoskeletal disorders was analysis from the cross-sectional studies ${ }^{14,18,29,32}$. Age showed a significant association with presence of musculoskeletal problems in low back, left shoulder, right shoulder, left hand, head, neck, TMJ, teeth/jaw, right and left fingers ${ }^{18,29}$. Older musicians suffered from physical problems more frequently than their younger colleagues. The percentage of physical problems increased with age $^{14}$. Older musicians were more likely to complain about their musculoskeletal symptoms ${ }^{32}$.

\section{Psychosocial factors:}

A positive association between stage fright or performance anxiety and musculoskeletal disorders was found in a multivariate analysis of two cross-sectional studies $^{11,29}$. Performance anxiety was a remarkable factor related to PRMD development in neck, shoulder, elbow and wrist $^{29}$.

\section{Health-related factors:}

Co-relation of cigarette smoking with musculoskeletal disorders was found in a univariate analysis, but papers performing multivariate analysis presented no such effect $^{31,37}$.

Work-related factors:

\begin{tabular}{|c|c|c|c|c|c|c|c|}
\hline \multicolumn{8}{|c|}{ Table 3.} \\
\hline $\begin{array}{l}\text { Sr } \\
\text { No. }\end{array}$ & $\begin{array}{l}\text { Author } \\
\text { Of The } \\
\text { Article }\end{array}$ & Title Of Article & $\begin{array}{l}\text { Name Of The } \\
\text { Journal }\end{array}$ & $\begin{array}{l}\text { Sample } \\
\text { Size }\end{array}$ & Age/Gender & $\begin{array}{l}\text { Type Of } \\
\text { Instrument } \\
\text { Played }\end{array}$ & $\begin{array}{l}\text { Result And Conclusion Of } \\
\text { Article }\end{array}$ \\
\hline 1. & $\begin{array}{l}\text { A. } \\
\text { Steinmetz } \\
\text { et.al } 2012\end{array}$ & $\begin{array}{l}\text { Playing-related } \\
\text { musculoskeletal } \\
\text { disorders in } \\
\text { music students- } \\
\text { associated } \\
\text { musculoskeletal } \\
\text { signs }\end{array}$ & $\begin{array}{l}\text { European } \\
\text { Journal of } \\
\text { Physical and } \\
\text { Rehabilitation } \\
\text { Medicine }\end{array}$ & $\mathrm{N}=55$ & $\begin{array}{l}18 \text { and above } \\
\text { years/ Both } \\
\text { genders }\end{array}$ & $\begin{array}{l}\text { All orchestral } \\
\text { musicians } \\
\text { (except viola and } \\
\text { tuba) }\end{array}$ & $\begin{array}{l}\text { This study reported } 81 \% \text { of } \\
\text { musicians experienced pain } \\
\text { and discomfort while playing } \\
\text { instruments. } 6.5 \% \text { indicated } \\
\text { pain to be 'almost always' } \\
\text { and } 34.4 \% \text { indicated pain to } \\
\text { occur 'very often'. } 18.8 \% \\
\text { indicated pain 'rarely' } \\
\text { associated with playing } \\
\text { instrument. In } 44.1 \% \\
\text { occurred during and after } \\
\text { playing an instrument, in } \\
28.6 \% \text { musicians pain } \\
\text { appeared within less of } 1 \\
\text { hour of playing an } \\
\text { instrument. Music students } \\
\text { were found to have more } \\
\text { positive findings in } \\
\text { musculoskeletal examination } \\
\text { than non-music student } \\
\text { group. }\end{array}$ \\
\hline
\end{tabular}


Ketaki Vijay Shinde et.al. Epidemiology of musculoskeletal disorders in musicians - systematic review.

\begin{tabular}{|c|c|c|c|c|c|c|c|}
\hline \multicolumn{8}{|c|}{ Table 3 Continued... } \\
\hline 2. & $\begin{array}{l}\text { Flavio M. } \\
\text { Silva, PT, } \\
\text { ScD et.al } \\
2018\end{array}$ & $\begin{array}{l}\text { Musicians injuries: } \\
\text { upper quarter motor } \\
\text { control deficits in } \\
\text { musicians with } \\
\text { prolonged } \\
\text { symptoms- a case } \\
\text { control study }\end{array}$ & $\begin{array}{l}\text { Musculoske } \\
\text { letal } \\
\text { Science and } \\
\text { Practice }\end{array}$ & $\mathrm{N}=81$ & $\begin{array}{l}\text { 18-65 years/ } \\
\text { both genders }\end{array}$ & $\begin{array}{ll}\text { Student } & \text { or } \\
\text { professional } & \\
\text { Instrumental } & \\
\text { musicians. } & \end{array}$ & $\begin{array}{l}\text { This study concludes that } \\
\text { musicians with prolonged } \\
\text { upper quarter playing related } \\
\text { pain presented with higher } \\
\text { prevalence of scapular } \\
\text { dyskinesis and lower } \\
\text { craniocervical flexion test } \\
\text { performance when compared } \\
\text { to musicians with no history } \\
\text { of prolonged upper quarter } \\
\text { playing related pain. }\end{array}$ \\
\hline 3. & $\begin{array}{l}\text { Dianna } \\
\text { Kenny } \\
\text { et.al } 2015\end{array}$ & $\begin{array}{l}\text { Performance-related } \\
\text { musculoskeletal } \\
\text { pain, depression and } \\
\text { music performance } \\
\text { anxiety in } \\
\text { professional } \\
\text { orchestral } \\
\text { musicians: a } \\
\text { population study }\end{array}$ & $\begin{array}{l}\text { Psychology } \\
\text { of Music }\end{array}$ & $\mathrm{N}=377$ & $\begin{array}{l}\text { 18-68 years/ } \\
\text { both genders }\end{array}$ & $\begin{array}{l}\text { Orchestra } \\
\text { musicians. }\end{array}$ & $\begin{array}{l}\text { This study concludes that } \\
318 \text { (84\%) musicians } \\
\text { reported pain interfering with } \\
\text { performance, } 100 \text { (26.7\%) } \\
\text { reported never experienced } \\
\text { PRMD, 184 (49.1\%) } \\
\text { musicians reported pain } \\
\text { rating between } 2 \text { and } 6,91 \\
(24 \%) \text { musicians reported } \\
\text { PRMD constantly. Female } \\
\text { musicians reported } \\
\text { significantly more frequent } \\
\text { occasions of pain interfering } \\
\text { with their performance. } \\
\text { Performance anxiety shows } \\
\text { positive association with } \\
\text { musculoskeletal disorders } \\
\text { development. }\end{array}$ \\
\hline 4. & $\begin{array}{l}\text { Filiz } \\
\text { Ozdemir } \\
\text { et.al } 2019\end{array}$ & $\begin{array}{l}\text { Evaluation of work- } \\
\text { related } \\
\text { musculoskeletal } \\
\text { disorders and } \\
\text { ergonomic risk } \\
\text { levels among } \\
\text { instrumentalist } \\
\text { musicians }\end{array}$ & $\begin{array}{l}\text { Annals of } \\
\text { Medical } \\
\text { Research }\end{array}$ & $\mathrm{N}=46$ & $\begin{array}{l}\text { 18-50 years/ } \\
\text { both genders }\end{array}$ & $\begin{array}{l}\text { Violinists, side } \\
\text { flutists, pianists } \\
\text { and balgama } \\
\text { players. }\end{array}$ & $\begin{array}{l}\text { This study found that most } \\
\text { common pain areas in any } \\
\text { period during lifetime were } \\
\text { found as hand-wrist ( } 65.2 \%) \text {, } \\
\text { neck ( } 58.8 \%) \text {, shoulders } \\
(52.3 \%) \text {. In last } 12 \text { months, } \\
\text { most frequent areas were } \\
\text { wrist ( } 50 \%) \text {, back ( } 43.5 \%) \\
\text { and neck ( } 39.7 \%) \text {. In last } \\
\text { month were hand-wrist } \\
(17.4 \%) \text {, neck ( } 17.7 \%) \text { and } \\
\text { waist (17.7\%). On the day of } \\
\text { evaluation, neck } 16.8 \% \text {, } \\
\text { shoulders } 12.8 \% \text { and lower } \\
\text { back } 11.2 \% \text { were reported. } \\
\text { Higher ergonomic risk level } \\
\text { was found among piano and } \\
\text { violin players. }\end{array}$ \\
\hline 5. & $\begin{array}{l}\text { Adedayo } \\
\text { T. } \\
\text { Ajidahun } \\
\text { et.al } 2017\end{array}$ & $\begin{array}{l}\text { Musculoskeletal } \\
\text { problems among } \\
\text { string } \\
\text { instrumentalist in } \\
\text { south africa }\end{array}$ & $\begin{array}{l}\text { South } \\
\text { African } \\
\text { Journal of } \\
\text { Physiothera } \\
\text { py }\end{array}$ & $\mathrm{N}=114$ & $\begin{array}{l}\text { 18-78 years/ } \\
\text { both genders }\end{array}$ & $\begin{array}{l}\text { String } \\
\text { instrumentalists }\end{array}$ & $\begin{array}{l}\text { This study concludes that } \\
\text { prevalence of } \\
\text { musculoskeletal problems } \\
\text { that affect the performance is } \\
\text { high among string } \\
\text { instrumentalists in South } \\
\text { Africa. }\end{array}$ \\
\hline 6. & $\begin{array}{l}\text { E. Savino } \\
\text { et.al } 2013\end{array}$ & \begin{tabular}{l}
\multicolumn{2}{l}{ Musculoskeletal } \\
disorders and \\
occupational stress \\
of violinists
\end{tabular} & $\begin{array}{l}\text { Journal of } \\
\text { Biological } \\
\text { Regulators } \\
\text { and } \\
\text { Homeostati } \\
\text { c Agents }\end{array}$ & $\mathrm{N}=12$ & $\begin{array}{l}23-26 \text { years/ } \\
\text { both genders }\end{array}$ & Violinists & $\begin{array}{l}\text { This study concludes that } \\
\text { painful symptoms related to } \\
\text { upper limbs were found to be } \\
83.3 \%, 33.30 \% \text { of lower } \\
\text { limbs, } 16.67 \% \text { had pain } \\
\text { related to jaw. Referred pain } \\
\text { in right or left shoulders or } \\
\text { both affected } 16.67 \% \text { and } \\
33.33 \% \text { subjects complained } \\
\text { about painful symptoms in } \\
\text { right elbow. }\end{array}$ \\
\hline 7. & $\begin{array}{l}\text { Paarup } \\
\text { et.al } 2011\end{array}$ & $\begin{array}{l}\text { Prevalence and } \\
\text { consequences of } \\
\text { musculoskeletal } \\
\text { symptoms in } \\
\text { symphony orchestra } \\
\text { musicians vary by } \\
\text { gender: a cross- } \\
\text { sectional study }\end{array}$ & $\begin{array}{l}\text { BioMed } \\
\text { Central }\end{array}$ & $\mathrm{N}=342$ & $\begin{array}{l}\text { 37-50 years/ } \\
\text { both genders }\end{array}$ & $\begin{array}{l}\text { Orchestra } \\
\text { musicians }\end{array}$ & $\begin{array}{l}\text { This study concludes high } \\
\text { annual prevalence of } 90 \% \text { for } \\
\text { PRMDs. Within the last year } \\
\text { most symphony orchestra } \\
\text { musicians experienced } \\
\text { musculoskeletal symptoms in } \\
\text { neck, back and upper } \\
\text { extremities. }\end{array}$ \\
\hline
\end{tabular}




\begin{tabular}{|c|c|c|c|c|c|c|c|}
\hline \multicolumn{8}{|c|}{ Table 3 Continued... } \\
\hline 8. & $\begin{array}{l}\text { Paarup } \\
\text { et.al } 2012\end{array}$ & 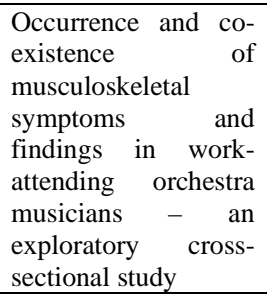 & $\begin{array}{l}\text { BioMed } \\
\text { Central }\end{array}$ & $\mathrm{N}=216$ & $\begin{array}{l}20-69 \\
\text { years/ both } \\
\text { genders }\end{array}$ & $\begin{array}{l}\text { Orchestra } \\
\text { musicians }\end{array}$ & $\begin{array}{l}\text { This study concludes that } \\
\text { Symptoms and findings both } \\
\text { were most common found in } \\
\text { back, neck and shoulders. }\end{array}$ \\
\hline 9. & $\begin{array}{l}\text { Katriina } \\
\text { Viljamaa } \\
\text { et.al } 2017\end{array}$ & \begin{tabular}{lr}
\multicolumn{2}{l}{ Musculoskeletal } \\
symptoms $\quad$ among \\
finnish & orchestral \\
musicians &
\end{tabular} & $\begin{array}{l}\text { Medical } \\
\text { Problem } \\
\text { s of } \\
\text { Performi } \\
\text { ng } \\
\text { Artists }\end{array}$ & $\mathrm{N}=361$ & $\begin{array}{l}5.0 \pm 10.0 \\
\text { years/ both } \\
\text { genders }\end{array}$ & $\begin{array}{l}\text { Orchestra } \\
\text { musicians }\end{array}$ & $\begin{array}{l}\text { This study concludes that } \\
\text { symphony orchestra musicians } \\
\text { experience nearly twice as much } \\
\text { as musculoskeletal symptoms of } \\
\text { the neck and upper extremities. }\end{array}$ \\
\hline 10. & 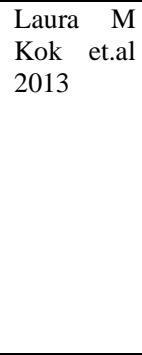 & $\begin{array}{l}\text { A comparative study } \\
\text { on the prevalence of } \\
\text { musculoskeletal } \\
\text { complaints among } \\
\text { musicians and non- } \\
\text { musicians }\end{array}$ & $\begin{array}{l}\text { BioMed } \\
\text { Central }\end{array}$ & $\begin{array}{l}\mathrm{N}=577 \\
(\mathrm{n}=83 \\
\text { music } \\
\text { students } \\
\mathrm{n}=494 \\
\text { medical } \\
\text { students) }\end{array}$ & $\begin{array}{l}\text { Music } \\
\text { students } \\
\text { mean age - } \\
21.5 \text { years } \\
\text { and } \\
\text { medical } \\
\text { students } \\
\text { mean age - } \\
22.1 \text { years/ } \\
\text { both } \\
\text { genders }\end{array}$ & $\begin{array}{l}\text { Classical } \\
\text { instrument } \\
\text { musicians }\end{array}$ & $\begin{array}{l}\text { This study concludes that } \\
\text { musculoskeletal complaints are } \\
\text { significantly more common } \\
\text { among musicians compared to } \\
\text { non-musicians with higher } \\
\text { number of upper extremity } \\
\text { complaints. }\end{array}$ \\
\hline 11. & $\begin{array}{l}\text { Dusica L. } \\
\text { Maric et.al } \\
2019\end{array}$ & $\begin{array}{l}\text { A painful symphony: } \\
\text { the presence of } \\
\text { overuse syndrome in } \\
\text { professional classical } \\
\text { musicians }\end{array}$ & $\begin{array}{l}\text { Internati } \\
\text { onal } \\
\text { Journal } \\
\text { of } \\
\text { Morphol } \\
\text { ogy }\end{array}$ & $\mathrm{N}=50$ & $\begin{array}{l}21-58 \\
\text { years/ both } \\
\text { genders }\end{array}$ & $\begin{array}{l}\text { Professional } \\
\text { classical } \\
\text { musicians }\end{array}$ & $\begin{array}{l}\text { This study concludes that high } \\
\text { prevalence of overuse syndrome } \\
\text { is found in professional classical } \\
\text { musicians. The upper back and } \\
\text { neck were the most frequent } \\
\text { symptom site for musicians and } \\
\text { primary symptom was pain. }\end{array}$ \\
\hline 12. & $\begin{array}{l}\text { Kaufman- } \\
\text { Cohen and } \\
\text { Ratzon } \\
\text { et.al } 2011\end{array}$ & $\begin{array}{l}\text { Correlation between } \\
\text { risk factors and } \\
\text { musculoskeltal } \\
\text { disorders among } \\
\text { classical musicians }\end{array}$ & $\begin{array}{l}\text { Occupati } \\
\text { onal } \\
\text { Medicin } \\
\text { e }\end{array}$ & $\mathrm{N}=59$ & $\begin{array}{l}26-66 \\
\text { years/ both } \\
\text { genders }\end{array}$ & $\begin{array}{l}\text { Classical } \\
\text { musicians }\end{array}$ & $\begin{array}{l}\text { This study concludes PRMD } \\
\text { annual prevalence of } 83 \% .73 \% \\
\text { musicians suffered from PRMDS } \\
\text { in more than one joint. } 58 \% \\
\text { reported upper extremity pain } \\
\text { during past year. High prevalence } \\
\text { of shoulder pain ( } 55 \%) \text {, lower } \\
\text { back pain ( } 49 \%) \text {, upper back pain } \\
(42 \%) \text { and neck pain ( } 39 \%) \text {. } \\
61 \% \text { of the string players } \\
\text { reported shoulder pain. The } \\
\text { RULA score was significantly } \\
\text { higher among string players in } \\
\text { comparison to wind players. }\end{array}$ \\
\hline 13. & $\begin{array}{l}\text { Steinmetz } \\
\text { et.al } 2015\end{array}$ & $\begin{array}{l}\text { Frequency, severity } \\
\text { and predictors of } \\
\text { playing-related } \\
\text { musculoskeletal pain } \\
\text { in professional } \\
\text { orchestral musicians } \\
\text { in germany }\end{array}$ & $\begin{array}{l}\text { Clin } \\
\text { Rheumat } \\
\text { ol }\end{array}$ & $\mathrm{N}=408$ & $\begin{array}{l}\text { Mean age - } \\
43.9 \text { years/ } \\
\text { both } \\
\text { genders }\end{array}$ & $\begin{array}{l}\text { Professional } \\
\text { orchestra } \\
\text { musicians }\end{array}$ & $\begin{array}{l}\text { This study concludes with } 89.5 \% \\
\text { lifetime prevalence of PRMDs in } \\
\text { musicians. String players, } \\
\text { particularly violinists, were the } \\
\text { most affected instrument group, } \\
\text { with } 50 \% \text { of musicians indicating } \\
\text { more than } 5 \text { pain regions. } \\
\text { Female sex and stage fright were } \\
\text { proven to be predictors of } \\
\text { musculoskeletal pain. }\end{array}$ \\
\hline 14. & $\begin{array}{l}\text { Adedayo } \\
\text { Tunde } \\
\text { Ajidahun } \\
\text { et.al } 2016\end{array}$ & $\begin{array}{lr}\text { Upper } & \text { extremity } \\
\text { disability } & \text { among } \\
\text { string } & \\
\text { instrumentalists- use } \\
\text { of the quick dash and } \\
\text { the ndi }\end{array}$ & $\begin{array}{l}\text { Congent } \\
\text { Medicin } \\
\text { e }\end{array}$ & $\mathrm{N}=99$ & $\begin{array}{l}33.3 \pm 15.3 \\
\text { years/ both } \\
\text { genders }\end{array}$ & $\begin{array}{l}\text { String } \\
\text { instrumentalist } \\
\mathrm{s}\end{array}$ & $\begin{array}{l}\text { This study concludes that } \\
\text { musculoskeletal problems were } \\
\text { reported by } 35 \text { ( } 35.7 \%) \\
\text { respondents (last } 7 \text { days) and } 56 \\
(56.6 \%) \text { reported symptoms of } \\
\text { musculoskeletal disorders over } \\
\text { the last year. } \\
\text { Musculoskeletal problems were } \\
\text { mainly reported in the low back } \\
(50.5 \%) \text { upper back ( } 49.5 \%) \text { and } \\
\text { neck ( } 46.5 \%) \text { and left shoulder } \\
(44.4 \%) \text {. } \\
\text { Problems were reported in four } \\
\text { or more body regions in the } \\
\text { upper extremity and trunk by } 39 \\
(39.4 \%) \text { of the string } \\
\text { instrumentalists. }\end{array}$ \\
\hline
\end{tabular}




\begin{tabular}{|c|c|c|c|c|c|c|c|}
\hline \multicolumn{8}{|c|}{ Table 3 Continued... } \\
\hline 15. & $\begin{array}{l}\text { Frederico } \\
\text { Baretto } \\
\text { Kochem } \\
\text { et.al } 2017\end{array}$ & $\begin{array}{l}\text { Prevalence and } \\
\text { associated factors of } \\
\text { playing-related } \\
\text { musculoskeletal } \\
\text { disorders in brazilian } \\
\text { violin players }\end{array}$ & $\begin{array}{l}\text { Medical } \\
\text { Problem } \\
\text { s of } \\
\text { Performi } \\
\text { ng } \\
\text { Artists }\end{array}$ & $\mathrm{N}=106$ & $\begin{array}{l}\text { Male mean } \\
\text { age }-24 \\
\text { years } \\
\text { Female } \\
\text { mean age - } \\
23 \text { years/ } \\
\text { both } \\
\text { genders }\end{array}$ & Violinists & $\begin{array}{l}\text { This study concludes that the } \\
\text { prevalence of PRMD among } \\
\text { Brazilian violin players is } \\
\text { alarmingly high both in last } 12 \\
\text { months ( } 86.8 \%) \text { and last } 7 \text { days } \\
\text { ( } 77.4 \%) \text { The most reported } \\
\text { painful regions were the neck, } \\
\text { thoracic spine and upper limbs. }\end{array}$ \\
\hline 16. & $\begin{array}{l}\text { Heiner } \\
\text { Gembris } \\
\text { et.al } 2017\end{array}$ & $\begin{array}{l}\text { Health problems of } \\
\text { orchestral musicians } \\
\text { from a life span } \\
\text { perspective: results } \\
\text { of a large-scale study }\end{array}$ & $\begin{array}{l}\text { Music \& } \\
\text { Science }\end{array}$ & $\mathrm{N}=2536$ & $\begin{array}{l}20-69 \\
\text { years/ both } \\
\text { genders }\end{array}$ & $\begin{array}{l}\text { Orchestra } \\
\text { musicians }\end{array}$ & $\begin{array}{l}\text { The study concludes that over } \\
\text { half of orchestral musicians } \\
(55 \%, \mathrm{n}=1,396) \text { had physical } \\
\text { problems that affected their } \\
\text { playing. The prevalence } \\
\text { increased significantly with } \\
\text { advancing age, and string players } \\
\text { and harpists had an above } \\
\text { average frequency of } \\
\text { experiencing physical problems. } \\
\text { Almost half of (49\%) orchestral } \\
\text { musicians stated they felt } \\
\text { pressure to perform. }\end{array}$ \\
\hline 17. & $\begin{array}{l}\text { Cláudia } \\
\text { Maria } \\
\text { SOUSA } \\
\text { et.al 2107 }\end{array}$ & $\begin{array}{l}\text { Playing-related } \\
\text { musculoskeletal } \\
\text { disorders } \\
\text { professional } \\
\text { orchestra musicians } \\
\text { from the north of } \\
\text { portugal: comparing } \\
\text { string and wind } \\
\text { musicians }\end{array}$ & $\begin{array}{l}\text { Acta } \\
\text { Med } \\
\text { Port }\end{array}$ & $\mathrm{N}=112$ & $\begin{array}{l}\text { Mean age } \\
37.8 \text { years/ } \\
\text { both } \\
\text { genders }\end{array}$ & $\begin{array}{l}\text { String and } \\
\text { wind } \\
\text { instrumentalist } \\
\mathrm{s}\end{array}$ & $\begin{array}{l}\text { This study concludes that } \\
\text { approximately two thirds of } \\
\text { professional orchestra musicians } \\
\text { from the North of Portugal suffer } \\
\text { from PRMD. Pain is more } \\
\text { prevalent in string players but } \\
\text { more intense in wind players. }\end{array}$ \\
\hline 18. & $\begin{array}{l}\text { Mark } \\
\text { Porter } \\
\text { et.al } 2018\end{array}$ & $\begin{array}{l}\text { Extent of playing- } \\
\text { related } \\
\text { musculoskeletal } \\
\text { problems in the irish } \\
\text { traditional music } \\
\text { community }\end{array}$ & $\begin{array}{l}\text { Medical } \\
\text { Problem } \\
\mathrm{s} \quad \text { of } \\
\text { Performi } \\
\text { ng } \\
\text { Artists }\end{array}$ & $\mathrm{N}=79$ & $\begin{array}{l}\text { Mean age } \\
35.38 \text { bers/ both } \\
\text { yearders } \\
\text { gender }\end{array}$ & Fiddle players & $\begin{array}{l}\text { This study concludes that } \\
\text { PRMDs are a problem for Irish } \\
\text { traditional fiddle players, } \\
\text { especially during times of intense } \\
\text { playing such as festivals. There } \\
\text { was a positive association found } \\
\text { between development of PRMDs } \\
\text { and increased hours of play. }\end{array}$ \\
\hline 19. & $\begin{array}{l}\text { R. Leaver } \\
\text { et.al } 2011\end{array}$ & $\begin{array}{l}\text { Musculoskeletal pain } \\
\text { in elite professional } \\
\text { musicians from } \\
\text { british symphony } \\
\text { orchestras }\end{array}$ & $\begin{array}{l}\text { Occupati } \\
\text { onal } \\
\text { Medicin } \\
\text { e }\end{array}$ & $\mathrm{N}=243$ & $\begin{array}{l}23-64 \\
\text { years/ both } \\
\text { genders }\end{array}$ & $\begin{array}{l}\text { Symphony } \\
\text { orchestra } \\
\text { musicians }\end{array}$ & $\begin{array}{l}86 \% \text { annual prevalence rate } \\
\text { affecting neck, low back and } \\
\text { shoulder. Weak association } \\
\text { found with psychosocial work } \\
\text { stressors and performance } \\
\text { anxiety. }\end{array}$ \\
\hline
\end{tabular}

Six papers studied the association between musculoskeletal disorders and the instruments ${ }^{13,22,28-30,36}$. Result of some studies present that upper string players are more likely to experience musculoskeletal symptoms when compared to other instruments $22,29,36$. A study reported a positive association between average weekly orchestra hours with number of symptomatic upper limb joints ${ }^{31}$. Biomechanical risk factors were found to be related to playing a musical instrument ${ }^{31}$. A paper presented a multivariate analysis which showed that number of affected areas and practice hours significantly elevates the disability scores for the general DASH scores and performing arts score ${ }^{30}$. A higher disability score is associated with an increase in the severity of musculoskeletal problem $^{30}$. A Quick Exposure Check (QEC) was carried out to determine the ergonomic risk level which was found higher among piano and violin players. Higher ergonomic risk levels were stated by musicians who reported low back pain and hip-thigh pain ${ }^{13}$.

\section{DISCUSSION}

Musicians are the workers who are exposed to several occupational hazards during their performance and practice period and are at high risk of developing Playing Related Musculoskeletal Disorders (PRMDs). The consequences of these injuries or disorders can lead to permanent performance loss or can even lead to premature termination of musical career $^{8 .}$ 
Though there is still a lack of publications with high methodological quality in the literature, 19 of 26 selected articles of moderate and high methodological quality were included in this review to study the epidemiology of musculoskeletal disorders in musicians.

The prevalence rate of PRMDs reported by musicians in last 12 months ranged from $86 \%$ to $90 \%$ in both genders 22,26,28,37. Leaver et.al carried out a survey study using questionnaire on 243 British orchestral musicians (51\% of 478 approached musicians) out of which $86 \%$ presented with musculoskeletal pain within the last 12 months. According to the findings of Kaufman-Cohen and Ratzon, who investigated the risk factors and musculoskeletal disorders in 59 classical orchestra musicians, $83 \%$ of the participating musicians reported symptoms in at least one body region within past 12 months. Kok et.al conducted a Dutch research study along with 83 orchestral musicians which presented with a prevalence rate for PRMD of $89.2 \%$ in the last 12 months indicating most affected anatomic regions neck, shoulders, elbows, wrists/hands and thoracic spine.

According to the studies few risk factors were found to have positive association with development of PRMDs. Dianna Kenny et.al conducted a population study on 377 orchestra musicians from Australia in which female musicians significantly reported more recurrent pain incidences that interfered with their performance. Compared to males, females had significantly higher susceptibility for having musculoskeletal symptoms Paarup et.al (2011). Kaufman-Cohen and Ratzon investigated the correlation between different risk factors and potential incidence of PRMD in classical musicians and found that female musicians report more musculoskeletal disorders than male musicians. Thus, female gender has proved to be a risk factor for development of PRMDs. Performance anxiety is positively related to development of musculoskeletal disorders according to a cross-sectional study carried out by A. Steinmetz et.al. This indicates positive association of musculoskeletal disorders and psychosocial factors.

The type of instrument played by a musician is a known risk factor for the development of musculoskeletal disorder. Adedayo T. Ajidahun et.al conducted a cross-sectional study among the string instrumentalists in South Africa and reported that $77 \%$ of string instrumentalists reported problems in one or more anatomic regions with $35 \%$ presently undergoing problems that are hindering with their performance. Musculoskeletal problems of severity ranging between mild to moderate were presented in this study. Similar results were found in a study conducted by Steinmetz et.al (2015) utilizing the numerical pain rating scale. A study was carried out in North Portugal by Claudia Maria SOUSA et.al comparing string and wind musicians with 112 professional orchestra musicians participating with results showing $62.5 \%$ of interviewed musicians suffer from acute PRMD with an average of from 5 to 3.8 VNS (verbal numeric scale). Results indicate that string players (67.1\%) are more affected than wind players $(54.1 \%)$ by PRMDs. Paarup et.al (2011) investigated from questionnaire data obtained from 342 professional musicians from Danish symphony orchestras and found a very high prevalence of musculoskeletal symptoms in neck, back and upper extremities among professional symphony orchestra musicians. The symptoms had extensive impact on musician's level of function in work with altered or impaired way of playing an instrument in both leisure as well as work time. Woodwind players were found to be having remarkably low risk for musculoskeletal problems as compared to other instrument groups.

The most frequently affected anatomic regions among string players were upper limbs and vertebral column. 61\% of musicians reported shoulder pain according 
to Kaufman-Cohen and Ratzon. Practice hours and amount of time spend playing an instrument presents positive association with the presence of musculoskeletal pain (Steinmetz et.al 2015). Kok et.al (2013) states that overuse syndrome is the maximum reported diagnosis amid the instrumental musicians experiencing PRMDs. Filiz Ozdemir et.al conducted a cross-sectional study including 46 instrumentalist musicians to evaluate the work-related musculoskeletal disorders and ergonomic risk levels and found high incidence of WMSDs and higher ergonomic risk levels among musicians.

\section{CONCLUSION}

Based on our analysis of included studies, most of the study stated high prevalence of Playing-Related Musculoskeletal Disorders among musicians. These disorders are associated with intrinsic factors like age, gender and psychosocial characteristics and extrinsic factors like work environment, practice hours and instrument type. The average PRMD prevalence among musicians ranges from $45 \%$ to $55 \%$. High prevalence of PRMDs is found majorly in string players.

\section{ACKNOWLEDGMENT}

The hands that are joined together to make this project a major success. It is indeed my privilege to express my sincere gratitude to principal Dr. A.P.J Abdul Kalam College of physiotherapy, Loni for his valuable advice and allowing me to carry out this project in this institution. I wish to express my great gratitude to my project guide Dr. Pradeep Borkar and all the teaching staff who have helped me to choose this project and provide me with constant guidance and support throughout the completion of this project I wish to thank all participants and school authorities for their cooperation and tolerance towards this project I would like to bow down to my parents, the almighty, sibling and my friends whose blessings, love and encouragement have always been a catalyst in all walks of my life .

Funding - No funding

Conflict Of Interest - All authors declare no potential conflict of interest related to this article.

Ethical Approval - Not Required

Registration

No.-

PIMS/DR.APJAKCOPT/IEC/2021/393

\section{REFERENCES}

1. Lederman RJ. Neuromuscular and musculoskeletal problems in instrumental musicians. Muscle \& Nerve: Official Journal of the American Association of Electrodiagnostic Medicine. 2003 May;27(5):549-61.

2. Brandfonbrener AG. Musculoskeletal problems of instrumental musicians. Hand clinics. 2003 May 1;19(2):231-9.

3. Zięba E, Zieliński G, Ginszt M. Etiology and epidemiology of playing-related musculoskeletal disorders-a systematic review. Journal of Education, Health and Sport. 2019 Jul 2;9(7):115-35.

4. Foxman I, Burgel BJ. Musician health and safety: Preventing playing-related musculoskeletal disorders. AAOHN journal. 2006 Jul;54(7):309-16.

5. Dommerholt J. Performing arts medicineinstrumentalist musicians Part I-General considerations. Journal of bodywork and movement therapies. 2009 Oct 1;13(4):3119.

6. Heinan M. A review of the unique injuries sustained by musicians. Journal of the American Academy of PAs. 2008 Apr 1;21(4):45-52.

7. Röijezon U, Berg J, Larsson A, Sundkvist P. Sitting postures' effects on movement behavior and musical performance among high string musicians-a pilot study. In Musicians' Health and Performance 3rd Conference 2018 (MHPC2018) 2018.study.

8. Zięba E, Zieliński G, Ginszt M. Etiology and epidemiology of playing-related musculoskeletal disorders-a systematic review. Journal of Education, Health and Sport. 2019 Jul 2;9(7):115-35.

9. Steinmetz A, Möller H, Seidel W, Rigotti T. Playing-related musculoskeletal disorders in music students-associated musculoskeletal 
signs. Eur J Phys Rehabil Med. 2012 Dec 1;48(4):625-33.

10. Gohil K, Sheth M, Vyas N. Prevalence of playing related musculoskeletal disorders in musicians. Int. J. Ther. Appl. 2016; 32:1003.

11. Kenny, D., \& Ackermann, B. (2015). Performance-related musculoskeletal pain, depression and music performance anxiety in professional orchestral musicians: A population study. Psychology of Music, 43(1), 43-60.

12. Lee HS, Park HY, Yoon JO, Kim JS, Chun JM, Aminata IW, Cho WJ, Jeon IH. Musicians' medicine: musculoskeletal problems in string players. Clinics in orthopedic surgery. 2013 Sep;5(3):155.

13. Ozdemir F, Tutus N, Akgun SO, Kilcik MH. Evaluation of work-related musculoskeletal disorders and ergonomic risk levels among instrumentalist musicians. Annals of Medical Research. 2019;26(11): 2630-4.

14. Gembris H, Heye A, Seifert A. Health problems of orchestral musicians from a life-span perspective: Results of a largescale study. Music \& Science. 2018 Jan 11; 1:2059204317739801.

15. Whiting P, Rutjes AW, Reitsma JB, et al. The development of QUADAS: a tool for the quality assessment of studies of diagnostic accuracy included in systematic reviews. BMC Med Res Methodol 2003; 3:25

16. Silva FM, Brismée JM, Sizer PS, Hooper TL, Robinson GE, Diamond AB. Musicians injuries: Upper quarter motor control deficits in musicians with prolonged symptoms-A case-control study. Musculoskeletal Science and Practice. 2018 Aug 1; 36:54-60.

17. Ling CY, Loo FC, Hamedon TR. Playingrelated musculoskeletal disorders among classical piano students at tertiary institutions in Malaysia: proportion and associated risk factors. Medical problems of performing artists. 2018 Jun 1;33(2):82-9.

18. Ajidahun AT, Mudzi W, Myezwa H, Wood WA. Musculoskeletal problems among string instrumentalists in South Africa. South African Journal of Physiotherapy. 2017 Feb 23;73(1):1-7.

19. Sathya P, D'souza H. A study to find prevalence of upper limb problems in Musicians. International Journal of Medical and Exercise Science, Vol 5 (4), 625-633 December 2019, DOI: 10.36678/ijmaes. 2019. v05i04. 001. 2019 May 4.

20. Vedpathak S, Haral P. Common musculoskeletal injuries faced by Indian drummers. Int J Health Sci Res. 2017; 7(5): 151-158..

21. Savino E, Iannelli S, Forcella L, Narciso L, Faraso G, Bonifaci G, Sannolo N. Musculoskeletal disorders and occupational stress of violinists. J Biol Regul Homeost Agents. 2013 Jul 1;27(3):853-9.

22. Paarup HM, Baelum J, Holm JW, Manniche C, Wedderkopp N. Prevalence and consequences of musculoskeletal symptoms in symphony orchestra musicians vary by gender: a cross-sectional study. BMC musculoskeletal disorders. 2011 Dec; 12(1):1-4.

23. Paarup HM, Baelum J, Manniche C, Holm JW, Wedderkopp N. Occurrence and coexistence of localized musculoskeletal symptoms and findings in work-attending orchestra musicians-an exploratory crosssectional study. BMC research notes. 2012 Dec;5(1):1-5.

24. Viljamaa K, Liira J, Kaakkola S, Savolainen A. Musculoskeletal symptoms among Finnish professional orchestra musicians. Medical problems of performing artists. 2017 Dec 1;32(4):195-200.

25. Pereira ÉF, Kothe F, Bleyer FT, Teixeira CS. Work-related stress and musculoskeletal complaints of orchestra musicians. Revista Dor. 2014 Apr; 15:1126.

26. Kok LM, Vlieland TP, Fiocco M, Nelissen RG. A comparative study on the prevalence of musculoskeletal complaints among musicians and non-musicians. BMC musculoskeletal disorders. 2013 Dec;14(1): 1-7.

27. Maric DL, Stojic M, Maric DL, Susnjevic S, Radosevic D, Knezi N, Knezi N. A painful symphony: the presence of overuse syndrome in professional classical musicians. International Journal of Morphology. 2019 Jul 1;37(3):1118-22.

28. Kaufman-Cohen Y, Ratzon NZ. Correlation between risk factors and musculoskeletal disorders among classical musicians. Occupational Medicine. 2011 Mar 1;61(2):90-5.

29. Steinmetz A, Scheffer I, Esmer E, Delank KS, Peroz I. Frequency, severity and 
predictors of playing-related musculoskeletal pain in professional orchestral musicians in Germany. Clinical rheumatology. 2015 May 1;34(5):965-73.

30. Ajidahun AT, Mudzi W, Myezwa H, Wood WA. Upper extremity disability among string instrumentalists-use of the quick DASH and the NDI. Cogent Medicine. 2016 Dec 31;3(1):1234535.

31. Kaufman-Cohen Y, Portnoy S, Sopher R, Mashiach L, Baruch-Halaf L, Ratzon NZ. The correlation between upper extremity musculoskeletal symptoms and joint kinematics, playing habits and hand span during playing among piano students. PloS one. 2018 Dec 19;13(12)e0208788.

32. Kochem FB, Silva JG. Prevalence and associated factors of playing-related musculoskeletal disorders in Brazilian violin players. Medical problems of performing artists. 2017 Mar 1;32(1):27-32.

33. Sousa CM, Machado JP, Greten HJ, Coimbra D. Playing-related musculoskeletal disorders of professional orchestra musicians from the north of Portugal: comparing string and wind musicians. Acta medica portuguesa. 2017 Apr 28;30(4):3026.
34. Kok LM, Haitjema S, Groenewegen KA, Rietveld $\mathrm{AB}$. The influence of a sudden increase in playing time on playing-related musculoskeletal complaints in high-level amateur musicians in a longitudinal cohort study. PLoS One. 2016 Sep 22; 11(9)e0163472.

35. Heredia L, Hinkamp D, Brodsky M, Llapur C. Playing-related problems among musicians of the Orquesta Buena Vista Social Club and supporting bands. Medical problems of performing artists. 2014 Jun 1;29(2):80-5.

36. Porter M, Wilson IM. Extent of playingrelated musculoskeletal problems in the Irish traditional music community: a survey. Medical problems of performing artists. 2018 Mar 1;33(1):47-55.

37. Leaver R, Harris EC, Palmer KT. Musculoskeletal pain in elite professional musicians from British symphony orchestras. Occupational Medicine. 2011 Dec 1;61(8):549-55.

How to cite this article: Shinde KV, Borkar P. Epidemiology of musculoskeletal disorders in musicians - systematic review. Int J Health Sci Res. 2021; 11(12): 114-127. DOI: https://doi. org/10.52403/ijhsr.20211217 